\title{
¿Qué responden los estudiantes del último año de educación secundaria, cuando en una situación fuera de contexto, se les pide escribir 10 oraciones que puedan deducirse de $3 x+5 y=10$ ?
}

\author{
Cecilia Barranguet, Consejo de Educación Secundaria, Uruguay
}

\begin{abstract}
Resumen: Este artículo tiene como objetivo difundir los resultados primarios de una encuesta realizada a 77 estudiantes del último nivel de educación secundaria en el marco de la educación pública de Uruguay, Argentina, Bolivia y Perú; los jóvenes entrevistados tienen entre 16 y 18 años de edad. Además, es una invitación a la comunidad de profesores de matemática a participar y continuar esta investigación con el fin de conocer las creencias de los estudiantes respecto a uno de los conceptos más trascendentes de la educación matemática como son las ecuaciones. Se pretende además sensibilizar en la urgencia de cuestionar y reformular nuestra práctica teniendo en cuenta los estudios académicos que se publican en torno al tema.
\end{abstract}

Palabras claves: matemática educativa, álgebra escolar, recta, ecuaciones

\begin{abstract}
The aim of this article is to spread the primary results of a survey carried out among 77 secondary school students in their last year of formal education (aged 16-18 years old) within the frame of state education in Uruguay, Argentina, Bolivia and Peru. In addition, this is an invitation for the community of teachers of math to participate and continue the investigation with the objective of getting to know the students' beliefs regarding one of the most transcendent concepts in the math education: equations. It is further expected to rise awareness of the urgency of questioning and reformulating our practice, bearing in mind the academic research papers published on the topic.
\end{abstract}

Keywords: Educational Math, School Algebra, Line, Equations

\section{Características de la investigación}

T a investigación es de tipo descriptivo de carácter mixto. La encuesta se ha aplicado a 77 estudiantes del último año de educación secundaria en el contexto de la educación formal y pública. Se ha solicitado a los directores de cada centro educativo que asignen un grupo en las condiciones de la población objetivo (estudiantes entre 16 y 18 años del último año de educación secundaria). Los resultados obtenidos en este análisis se refieren únicamente a la población muestreada.

La encuesta fue aplicada en el año 2014 por quien escribe, en 4 centros educativos de Montevideo (Uruguay), Iruya (Argentina), La Paz (Bolivia) y Lima (Perú). Todos los centros educativos involucrados son céntricos y fueron elegidos en forma aleatoria. Una vez que el director del centro educativo asigna el grupo (según conveniencia de horarios), se aplica la encuesta.

La estructura de la encuesta consta de una sola consigna que es la siguiente: "Escribir diez oraciones que puedan deducirse de: $3 x+5 y=10 "$.

Con esta consigna, se apela a la palabra que pueda surgir del estudiante, a la formulación de oraciones. Se utiliza a la misma como vehículo para acceder al pensamiento de los estudiantes, a través del cual, expresarán los conceptos que consideren pertinentes; como expresa Papini, "Una palabra es una generalización, se refiere a una clase de objeto y no a un solo objeto y obligatoriamente tiene significado (no puede ser un sonido vacío). Por lo tanto, la palabra es un acto verbal del pensamiento, que refleja la realidad en un sentido distinto del que lo hacen la percepción y la sensación." (Papini 2003: 55). Debido a esto, resultaría interesante realizar la misma encuesta a los "expertos" o a estudiantes universitarios o a estudiantes del profesorado de matemática para observar en

Revista Internacional de Aprendizaje en Ciencia, Matemáticas y Tecnología

Volumen 1, Número 1, 2014, <http://sobrelaeducacion.com>, ISSN 2386-8791

(C) Global Knowledge Academics. Cecilia Barranguet. Todos los derechos

reservados. Permisos: soporte@gkacademics.com 
qué modo varían los conceptos asociados y la capacidad de formular oraciones que abarquen conceptos de mayor complejidad.

Para que los estudiantes comprendan más la consigna, se dibuja un triángulo en el pizarrón y se hace una lluvia de ideas de las oraciones que se podrían escribir respecto a ese objeto matemático. Por ejemplo: es un triángulo, tiene 3 lados, tiene 3 vértices, se le puede calcular el área, su área se calcula multiplicando la base por la altura y dividiendo entre 2, etc. Luego, se les presenta la consigna y durante 10 minutos, en una hoja y en silencio, cada estudiante escribe lo que considera pertinente.

\section{Antecedentes que motivan la investigación}

Desde temprana edad los estudiantes de educación primaria trabajan con ecuaciones en actividades que solicitan completar expresiones parecidas a $5+\ldots=18$. También en resolución de problemas del estilo "Juan compró 3 caramelos del mismo precio y gastó $\$ 6$, ¿cuál fue el precio de cada caramelo?" Luego, por lo general en los primeros años de educación secundaria, se formalizan las expresiones algebraicas y se comienza a trabajar con ecuaciones propiamente dichas. Más adelante, se trabaja con ecuaciones "lineales" con dos variables, luego con sistemas de ecuaciones "lineales", con funciones polinómicas de primer y segundo grado y expresiones algebraicas, ecuaciones y funciones más complejas. Sin embargo, los estudiantes que ingresan a la universidad continúan teniendo dificultades con el manejo de las ecuaciones y las funciones.

Varias investigaciones analizan la dificultad de los estudiantes en la manipulación y la comprensión de las ecuaciones. Los problemas detectados son diversos, entre ellos están la dificultad de: resolver ecuaciones, traducir del lenguaje natural al lenguaje matemático y viceversa (Cardona, 2007; Londoño et al., 2011), aplicar ecuaciones a la resolución de problemas (Londoño et al., 2011; Palarea, 1998), interpretar el conjunto solución de una ecuación (Oaxaca, 2003; Ochoviet, 2005), vincular el conjunto solución de la ecuación con su representación gráfica (Oaxaca, 2003; Panizza et al., 1999), interpretar que la ecuación permite hallar cada uno de los puntos de una figura geométrica (Panizza et al., 1999) etc. En particular, este estudio se concentra en las últimas tres dificultades mencionadas.

Por lo anteriormente expuesto, es que surge la motivación para investigar qué es lo que subyace del conocimiento que tienen los estudiantes sobre las ecuaciones, no a través de una propuesta clásica en las clases de matemáticas, no a través de la manipulación con ecuaciones, no a través de la resolución de problemas, no a través de la aplicación de una secuencia didáctica, no a través de la entrevista, sino a través de una situación totalmente descontextualizada y abstracta (por la característica de la consigna y por la forma en que se aplicó).

Otra motivación para investigar sobre el tema, es la constante percepción de que el conjunto solución de una ecuación y los elementos de una figura geométrica, no tienden a estar asociados en la inmensa mayoría de los estudiantes. Esta percepción, es también expresada en el artículo de Panizza et al. (1999:467) en el párrafo siguiente: "Por otra parte, cualquiera haya sido el trabajo realizado alrededor de "ecuación de la recta", éste no parece suficiente para que los alumnos puedan establecer una relación entre los puntos de la recta y las soluciones de la ecuación correspondiente." En mi opinión, extiendo la afirmación anterior, no solamente a las rectas, sino a cualquier otra figura geométrica que se le representa a través de una ecuación. De todos modos, sería inútil sostener esa percepción, pues nos adherimos a la afirmación de que "La resolución de tareas que ponen en juego diferentes modos de pensamiento contribuye a desarrollar un mejor entendimiento del concepto y posibilita que el estudiante pueda recurrir a diferentes heurísticas para responder a las situaciones" (Ochoviet 2009: 268).

Por lo tanto, el objetivo de esta investigación es analizar qué es lo que los estudiantes del último año de educación secundaria pueden plasmar al enfrentarse a la consigna: "escribir 10 oraciones que puedan deducirse de $5 x+3 y=10$ ", por otro lado, se pretende indagar si, en una situación fuera de contexto, los estudiantes pueden expresar conceptos que permitan acompañar (o no) la hipótesis de que los estudiantes no vinculan este tipo de ecuaciones con el conjunto solución de la misma ni con la figura que se obtiene en su representación gráfica. 


\section{Análisis de los resultados}

El 49,4\% de los estudiantes escribió 6 o menos oraciones pero no fue por falta de tiempo sino por falta de "ideas". En total se escribieron 490 oraciones.

Del análisis de las oraciones se desprenden once categorías que se detallan a continuación: tiene dos incógnitas ${ }^{1}$ (o variables), es una ecuación, hace referencia a la ecuación de la recta, hace referencia al procedimiento de resolución, llamarle ejercicio o problema, hace referencia a los elementos del conjunto solución y/o puntos, hace referencia a los sistemas de ecuaciones, hace referencia a la representación gráfica, es de carácter descriptivo que no contempla las dos primeras categorías, hace referencia a problemas de aplicación y por último es una expresión de sentimiento.

En la siguiente tabla se muestra la distribución de las respuestas según la categoría especificada en cada columna. Cuando la cantidad de oraciones no coincide con la cantidad de estudiantes, es porque hay estudiantes que escribieron más de una oración referida a una misma categoría.

Tabla 1: Distribución de respuestas por categoría

\begin{tabular}{|l|c|c|c|c|}
\hline & $\begin{array}{c}\text { Cantidad } \\
\text { de } \\
\text { oraciones }\end{array}$ & $\begin{array}{c}\text { Cantidad } \\
\text { de } \\
\text { estudiantes }\end{array}$ & $\begin{array}{c}\text { Porcentaje } \\
\text { de } \\
\text { oraciones }\end{array}$ & $\begin{array}{c}\text { Porcentaje } \\
\text { de } \\
\text { estudiantes }\end{array}$ \\
\hline Tiene dos incógnitas & 58 & 58 & 11,4 & 75,3 \\
\hline Es una ecuación & 62 & 62 & 13,2 & 80,5 \\
\hline Referencia a la ecuación de la recta & 15 & 12 & 3,2 & 15,6 \\
\hline Referencia al procedimiento de resolución & 58 & 36 & 12,4 & 46,8 \\
\hline Llamarla ejercicio o problema & 22 & 21 & 4,7 & 27,3 \\
\hline Referencia a elementos del conjunto solución y/o puntos & 60 & 33 & 12,8 & 42,9 \\
\hline Referencia a sistemas de ecuaciones & 12 & 8 & 2,6 & 10,4 \\
\hline Referencia a la representación gráfica & 23 & 13 & 4,9 & 16,9 \\
\hline De carácter descriptivo & 136 & 32 & 29,1 & 41,6 \\
\hline Referencia a problemas de aplicación & 7 & 7 & 1,5 & 9,1 \\
\hline Expresión de sentimiento & 15 & 12 & 3,2 & 15,6 \\
\hline
\end{tabular}

Fuente: Elaboración propia, 2014.

Se puede ver que más del $75 \%$ de los estudiantes reconocen que es una ecuación y que tiene dos incógnitas o variables.

El mayor porcentaje de las oraciones es de carácter descriptivo (26,6\%), pero si además consideramos que las dos primeras categorías $(23,4 \%)$ también son de carácter descriptivo, obtenemos que el $50 \%$ de las oraciones son de carácter descriptivo. Son ejemplos de oraciones de esta categoría: "Se divide en dos términos", "los números 3, 5 y 10 son llamados coeficientes", "los números sumados son enteros".

Las oraciones pertenecientes a la categoría en que se hace referencia al procedimiento de resolución ( $11,3 \%$ de la cantidad de oraciones y 46,8\% de los estudiantes), lo hacen de un modo muy vago. En algunos casos se apela a la transformación de la ecuación en ecuaciones equivalentes, pero ningún estudiante utiliza la palabra equivalente. Ejemplos de oraciones de esta categoría: "Primero se debe sacar el valor de x para poder llegar a descubrir y", "Se resuelve aplicando propiedades", "Se puede hallar y despejando", "Se puede resolver mediante tabla de valores".

Es relevante que el $42,9 \%$ de los estudiantes hace referencia a elementos del conjunto solución o a los puntos asociados a dichos elementos, sin embargo, un análisis más detallado de las respuestas nos permite observar que en su gran mayoría, las referencias son a un solo punto de la recta y/o a un solo elemento del conjunto solución, pero esa referencia también es vaga, ya que solo un estudiante encuestado $(1,3 \%)$ escribió dos elementos del conjunto solución de la ecuación y otro estudiante escribió sólo uno. El resto no escribió ninguno, aunque algunos hacen alusión a cómo se

\footnotetext{
${ }^{1}$ Por diversos motivos en este análisis no se distingue si el alumno ha hecho referencia a las incógnitas o variables.
} 
hallarían. Ejemplos de esta categoría son: "Podemos saber el valor de las incógnitas", "Es una ecuación para calcular el valor de x y de y", "Se tiene que dar una resolución o solución", "No se puede resolver", "Tiene dos respuestas para cada variable".

De lo anterior se puede sospechar que quizás los estudiantes no han escrito elementos del conjunto solución debido a que no les parece que esto sea una oración o que sea una oración relevante, sin embargo, si lo vinculamos al estudio de Panizza et al. (1999:456) no debería llamarnos la atención de que el motivo se deba a la poca comprensión de la solución de la ecuación ya que, según expresan en su artículo: "Se les solicitaba que propusieran una solución de la ecuación $3 x+2 y=7$. El $90 \%$ de los estudiantes no pudo obtener ninguna solución de la ecuación. El 10\% restante utilizó un procedimiento sorprendente para nosotras: agregar otra ecuación lineal y resolver el sistema resultante." Esto último también se vio reflejado en esta investigación, ya que el 10,4\% de los estudiantes hace referencia a los sistemas de ecuaciones.

Solo el 15,6\% de los estudiantes hace referencia a la ecuación de la recta.

Las oraciones que hacen referencia a la recta aparecen detalladas a continuación. En esta tabla también se puede observar la tendencia de ciertos grupos a hacer referencia a ciertos conceptos, en este caso en particular, solo estudiantes de los grupos $\mathrm{b} \mathrm{y} \mathrm{u}^{2}$.

Tabla 2: Oraciones de la categoría "referencia a la ecuación de la recta"

\begin{tabular}{|l|l|l|l|}
\hline $\mathrm{b}$ & 24 & 10 & Es la ecuación de una línea recta \\
\hline $\mathrm{b}$ & 26 & 9 & Se lo puede usar en líneas paralelas \\
\hline $\mathrm{b}$ & 26 & 9 & También en las perpendiculares \\
\hline $\mathrm{b}$ & 26 & 9 & Se puede usar entre un punto dado y una recta \\
\hline $\mathrm{b}$ & 27 & 10 & Es la ecuación de una recta \\
\hline $\mathrm{b}$ & 34 & 6 & Se puede representar en una recta \\
\hline $\mathrm{b}$ & 40 & 10 & Es una recta en el eje cartesiano \\
\hline $\mathrm{b}$ & 44 & 10 & Es la ecuación de una línea recta \\
\hline $\mathrm{b}$ & 45 & 8 & Se lo puede interpretar como una recta \\
\hline $\mathrm{b}$ & 45 & 8 & Se puede encontrar la pendiente m \\
\hline $\mathrm{b}$ & 49 & 8 & Esta expresión puede figurar una recta \\
\hline $\mathrm{b}$ & 50 & 7 & Es una recta en el eje cartesiano \\
\hline $\mathrm{u}$ & 72 & 4 & Esuna recta. \\
\hline $\mathrm{u}$ & 73 & 2 & Esuna recta. \\
\hline $\mathrm{u}$ & 75 & 2 & Representa una recta, -10 es el término independiente, por lo tanto la ordenada enelorigen. \\
\hline
\end{tabular}

Fuente: Elaboración propia, 2014.

Otra forma de analizar las respuestas fue según el uso de palabras, que por su relevancia, se consideraron claves para responder a nuestras preguntas: punto, recta, infinito, par (por par ordenado) y conjunto (por conjunto solución).

Se observa que:

Ningún estudiante utilizó la palabra infinito; palabra que, utilizada correctamente, ayudaría a apreciar una mayor comprensión de la ecuación.

En la siguiente tabla se transcriben las únicas oraciones en las que aparece la palabra punto.

\footnotetext{
${ }^{2}$ En la primera columna se encuentra el grupo al que pertenece el alumno (Argentina (a), Bolivia (b), Perú (u) y Uruguay (u)) en la segunda columna el número asignado al alumno, en la tercera columna la cantidad de oraciones que formula dicho alumno y en la cuarta columna la oración.
} 
Tabla 3: Oraciones que contienen la palabra "punto"

\begin{tabular}{|l|l|l|l|}
\hline $\mathrm{b}$ & 26 & 9 & Se puede usar entre un punto dado y una recta \\
\hline $\mathrm{b}$ & 28 & 9 & Podría ser un punto en el plano cartesiano \\
\hline $\mathrm{b}$ & 32 & 10 & Se usa las variantes para hallar puntos \\
\hline $\mathrm{b}$ & 32 & 10 & Las variantes x e y son puntos para traspasar en un plano cartesiano \\
\hline $\mathrm{b}$ & 36 & 8 & Se halla puntos o coordenadas \\
\hline $\mathrm{b}$ & 37 & 10 & Para hallar las coordenadas de un punto a otro \\
\hline $\mathrm{b}$ & 37 & 10 & Hallar el punto de una figura geométrica \\
\hline $\mathrm{b}$ & 38 & 10 & Es una fórmula que sirve para hallar los puntos \\
\hline $\mathrm{b}$ & 38 & 10 & Son puntos que se lleva al plano cartesiano \\
\hline $\mathrm{b}$ & 40 & 10 & Es el resultado de dos puntos \\
\hline $\mathrm{b}$ & 45 & 8 & Nos puede indicar un punto con sus coordenadas $\mathrm{P}(\mathrm{x}, \mathrm{y})$ \\
\hline $\mathrm{b}$ & 50 & 7 & Es el resultado de dos puntos \\
\hline $\mathrm{u}$ & 77 & 9 & Para hallarlo tendríamos que tomar dos puntos y despejar . \\
\hline
\end{tabular}

Fuente: Elaboración propia, 2014.

Por otro lado, si filtramos las oraciones según el uso de la palabra solución, obtenemos solamente 5 oraciones, de las cuales dos corresponden al estudiante 32, otras dos al estudiante 37 y la última al estudiante 38 , todos ellos del centro educativo b. Por lo que solo 3 estudiantes utilizan la palabra solución (o resolución) en sus oraciones, lo que significa el 3,9\% de los estudiantes.

Tabla 4: Oraciones que contienen la palabra "solución"

\begin{tabular}{|l|l|l|l|}
\hline $\mathrm{b}$ & 32 & 10 & Se tiene que dar una resolución o solución \\
\hline $\mathrm{b}$ & 32 & 10 & Un problema que tiene solución \\
\hline $\mathrm{b}$ & 37 & 10 & Tiene una resolución de $\mathrm{x}$ e y \\
\hline $\mathrm{b}$ & 37 & 10 & Tiene una solución fácil \\
\hline $\mathrm{b}$ & 38 & 10 & Es una ecuación que se utiliza para dar solución \\
\hline
\end{tabular}

Fuente: Elaboración propia, 2014.

Solamente un estudiante del total de encuestados utiliza la palabra conjunto y la oración que escribió fue: "es un conjunto de ecuaciones", lo que hace sospechar que se está refiriendo al sistema de ecuaciones, pero no al conjunto solución de la ecuación.

\section{Conclusiones y consideraciones}

La mayoría de las oraciones coinciden con las esperadas. O sea, utilizan muchas oraciones que describen la ecuación, pero no profundizan en el significado de la misma, siendo muy poco precisas las oraciones en las que el estudiante intenta referirse a la solución y a la interpretación de la misma.

Las oraciones generadas por los estudiantes no permiten observar un vínculo relevante entre la ecuación planteada y el concepto de punto, recta, solución, infinito y conjunto solución.

No se puede observar (quizás debido al instrumento utilizado) que los estudiantes manejen con soltura la forma en que se hallan los elementos del conjunto solución de una ecuación con dos variables, debido a que solo dos estudiantes lo realizaron.

No hay evidencias de que los estudiantes vinculen los infinitos puntos de la recta con los infinitos elementos de la solución de la ecuación. Esto puede deberse a que los estudiantes no interpretan que pueden escribir oraciones vinculadas con dichos conceptos o a que los docentes de los estudiantes entrevistados no hayan trabajado haciendo hincapié en la relación de los conceptos elegidos como palabras claves. De todos modos, los resultados obtenidos, son totalmente coherentes con los obtenidos en el trabajo citado de Panizza et al. (1999).

Los resultados primarios de esta investigación nos conducen una vez más a pensar que estamos frente a un problema muy complejo en el que aún quedan muchas cosas por hacer. A la vez, surgen 
nuevas preguntas como ¿por qué los estudiantes no utilizan la palabra infinito en sus oraciones?, ¿por qué no escriben elementos del conjunto solución?, ¿qué metodologías están contribuyendo a que estas dificultades aparezcan comúnmente entre los estudiantes?, ¿son la enseñanza de los sistemas de ecuaciones y la reiterada aplicación de ecuaciones a la resolución de problemas de solución única, un obstáculo epistemológico para la comprensión de la infinidad de elementos del conjunto solución de una ecuación?

Como última apreciación, se invita a los lectores a la lectura sistemática de los estudios vinculados con el tema y a tener en cuenta las consideraciones de los autores que proponen estrategias para la consolidación de aprendizajes significativos. 


\section{REFERENCIAS}

Cardona, M. A. (2007). Desarrollando el pensamiento algebraico en alumnos de octavo grado del CIIE a través de la resolución de problemas (Tesis de Maestría). Universidad Pedagógica Nacional Francisco Morazán. Disponible en: http://www.cervantesvirtual.com/obra/ desarrollando-el-pensamiento-algebraico-en-alumnos-de-octavo-grado-del-ciie-a-traves-dela-resolucion-de-problemas/ (12/08/2014)

Duval, R. (1998). Registros de representación semiótica y funcionamiento cognitivo del pensamiento. En F. Hitt (Ed.), Investigaciones en Matemática Educativa II (pp. 173-201). México: Grupo Editorial Iberoamérica.

- (2002). Semiosis y pensamiento humano. Registros semióticos y aprendizajes intelectuales. Cali: Ed. Universidad del Valle.

Figueroa, R. (2013). Resolución de problemas con sistemas de ecuaciones lineales con dos variables. Una propuesta para el cuarto año de secundaria desde la teoría de situaciones didácticas. Disponible en: http://m.tesis.pucp.edu.pe/repositorio/handle/123456789/4736 $(14 / 08 / 2014)$

Martínez, G. (2005). Los procesos de convención matemática como generadores de conocimiento. Relime, 8(2), pp. 195-218.

Gómez, E. (2008). La construcción de la noción de variable. www.matedu.cicata.ipn.mx/tesis/ doctorado/Gomez_2008.pdf (22/11/2014)

Londoño, S., Muñoz, L., Jaramillo, C. y Villa, J. (2011). Una aproximación a la noción de ecuación lineal. Disponible en: http://www.etnomatematica.org/publica/articulos/Una\%20 aproximaci\%C3\%B3n\%20a\%201a\%20noci\%C3\%B3n\%20de\%20ecuaci\%C3\%B3n\%20lin eal.pdf $(21 / 11 / 2014)$

Oaxaca, J., De la Cruz, J. y Sánchez, J. (2003). Dificultades en el tránsito del razonamiento sintéticogeométrico al analítico- aritmético en la solución de sistemas de ecuaciones lineales. dcb.fic.unam.mx/Eventos/ForoMatematicas2/memorias2/ponencias/41.pdf(22/11/2014)

Ochoviet, C. (2005). ¿A.B=0_A=0Ú $B=0$ ? Reflexiones e implicaciones en la Enseñanza de la matemática (Tesis de Maestría). Disponible en: www.clame.org.mx/documentos/alme\%2017.pdf (14 /08/2014)

- (2009). Sobre el concepto de solución de un sistema de ecuaciones lineales con dos incógnitas. www.matedu.cicata.ipn.mx/tesis/doctorado/ochoviet_2009.pdf (12/08/2014)

Palarea, M. (1998). La adquisición del lenguaje algebraico y la detección de errores comunes cometidos en el álgebra por alumnos de 12 a 14 años (Tesis doctoral). Universidad de la Laguna, España. Disponible en: ftp://tesis.bbtk.ull.es/ccppytec/cp90.pdf (21/11/2014)

Panizza M., Sadovsky P., Sessa C. (1999). La ecuación lineal con dos variables: entre la unicidad y el infinito. Enseñanza de la Ciencias, 17(3), pp. 453-461.

Papini, M. (2003). Algunas explicaciones Vigotskianas para las primeras aproximaciones del álgebra. Revista latinoamericana de investigación en matemática educativa, 6(001), pp. 41-71.

Pereira, Z. (2011). Los diseños de método mixto en la investigación en educación: Una experiencia concreta. Revista electrónica Educare, XV,(1). Disponible en: www.redalyc.org/pdf/1941/ 194118804003.pdf (15/08/2014).

Ursini, S. (1996). Creación de un potencial para trabajar con la noción de variable. México: Grupo editorial Iberoamérica. 


\section{SOBRE LA AUTORA}

Cecilia Barranguet: Egresada del Instituto de Profesores Artigas de Uruguay en el 2001. Trabaja como profesora de matemática en el Consejo de Educación Secundaria en dicho país, como profesora adscriptora de estudiantes de profesorado y en PLEMUU (Plenario de la Mujer del Uruguay), como educadora en contexto crítico. Diploma en Educación con Énfasis en Didáctica de la Matemática en la Universidad Católica del Uruguay. 